\title{
Let's talk about 'chemsex': Sexualized drug use among men who have sex with men
}

\author{
Christopher L. Clayton, MD, EdM, José Sánchez-Cruz, MD, and Gemma D. Espejo, MD
}

Dr. Clayton is a PGY-3 Psychiatry Resident, NYU Grossman School of Medicine, New York, New York. Dr. Sánchez-Cruz is a PGY-3 Psychiatry Resident, NYU Grossman School of Medicine, New York, New York. Dr. Espejo is Assistant Professor, Albert Einstein College of Medicine, and Attending Psychiatrist, Montefiore Hospital, Bronx, New York.

\section{Disclosures}

The authors report no financial relationships with any companies whose products are mentioned in this article, or with manufacturers of competing products.

doi: 10.12788/cp.0196

\section{$f$}

Discuss this article at www.facebook.com/ MDedgePsychiatry (
$\Gamma$ onsider the following patients who have presented to our hospital system:

- A 27-year-old gay man is brought to the emergency department by police after bizarre behavior in a hotel. He is paranoid, disorganized, and responding to internal stimuli. $\mathrm{He}$ admits to using methamphetamine before a potential "hookup" at the hotel

- A 35-year-old bisexual man presents to the psychiatric emergency department, worried he will lose his job and relationship after downloading a dating app on his work phone to buy methamphetamine

- A 30-year-old gay man divulges to his psychiatrist that he is insecure about his sexual performance and intimacy with his partner because most of their sexual contact involves using gamma-hydroxybutyric acid (GHB).

These are just some of the many psychiatric presentations we have encountered involving "chemsex" among men who have sex with men (MSM).

\section{What is 'chemsex?'}

"Chemsex" refers to the use of specific drugs-mainly methamphetamine, mephedrone, or GHB-before or during sex to reduce sexual disinhibitions and to facili-

Every issue of CURRent Psychiatry has its 'Pearls'

Yours could be found here.

Read the 'Pearls' guidelines for manuscript submission at MDedge.com/ CurrentPsychiatry/page/pearls. Then, share with your peers a 'Pearl' of wisdom from your practice. tate, initiate, prolong, sustain, and intensify the encounter. ${ }^{1}$ Chemsex participants report desired enhancements in:

- confidence and ability to engage with partners

- emotional awareness and shared experience with partners

- sexual performance and intensity of sensations. ${ }^{1}$

\section{How prevalent is it?}

Emerging in urban centers as a part of gay nightlife, chemsex has become increasingly prevalent among young MSM, fueled by a worldwide rise in methamphetamine use. ${ }^{1,2}$ In a large 2019 systematic review, Maxwell et $\mathrm{al}^{1}$ reported a wide range of chemsex prevalence estimates among MSM (3\% to $29 \%$ ). Higher estimates emerged from studies recruiting participants from sexual health clinics and through phone-based dating apps, while lower estimates tended to come from more representative samples of MSM. In studies from the United States, the prevalence of chemsex ranged from $9 \%$ to $10 \%$ in samples recruited from gay pride events, gay nightlife venues, and internet surveys. Across studies, MSM participating in chemsex were more likely to identify as gay, with mean ages ranging from 32 to 42 years, and were more likely to be HIV-positive. ${ }^{1}$

Methamphetamine was the most popular drug used, with GHB having higher prevalence in Western Europe, and mephedrone more common in the United Kingdom. ${ }^{1}$ Injection drug use was only examined in studies from the United Kingdom, the Netherlands, and Australia and showed a lower overall prevalence rate- $1 \%$ to $9 \%$. 


\section{Potential mental health sequelae of chemsex}

\begin{tabular}{|l|}
\hline Acute \\
\hline Substance-induced psychosis, including agitation and paranoia \\
\hline Substance-induced mood disorders \\
\hline Drug overdose (with concern for mixed or laced substances) \\
\hline Acute stress: sexual assault, sexually transmitted infection exposure or diagnosis \\
\hline Chronic \\
\hline Substance use disorders \\
\hline Psychotic disorders \\
\hline Mood or anxiety disorders \\
\hline $\begin{array}{l}\text { Psychosocial stressors: job instability, legal problems, issues with intimacy and loneliness, stigma of } \\
\text { being a chemsex user, strains in social networks and social isolation }\end{array}$ \\
\hline
\end{tabular}

\section{Table 2}

\section{Tips for helping patients who engage in chemsex}

1. Screen for chemsex. Approach patients in a curious, nonjudgmental, sex-positive manner, because MSM often face stigma and discrimination due to their sexual practices. You may choose to start with a normalizing statement such as "many people take substances to enhance sexual experiences."

Screening questions include:

- Have you used drugs to enhance your sex life?

- Are these drugs a part of your sex life?

Follow-up questions include:

- What do these drugs do for your sex life?

- How do you like to use these substances?

- Who do you like to use them with?

- Is there anything you don't like about your drug use?

2. Emphasize harm reduction, rather than abstinence only, working to meet patients where they are to minimize potential harms of chemsex.

- Open a dialogue about what patients enjoy about chemsex, while educating them about the risks of different sexual practices, including sex with multiple partners.

- Offer STI screening, and counsel patients on condom use and HIV pre- or post-exposure prophylaxis.

- Offer naloxone kits and training to prevent overdose deaths from opiates, which may be unknowingly mixed with, or used in conjunction with, chemsex drugs.

3. Treat substance use disorders and comorbid mental health conditions, while remaining attentive to the role sex plays in their presentation and recovery.

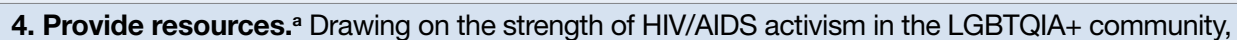
many groups have emerged to provide education, harm reduction, and support for those who engage in chemsex.

- Crystal Meth Anonymous (www.crystalmeth.org) has many LGBTQIA+-specific resources and a 24-hour helpline: 1-855-METH-FREE (1-855-638-4373).

- Organizations such as Seeking Integrity (www.seekingintegrity.com/chemsex-gay-and-

bisexual-men) offer treatment for chemsex addiction.

- General substance use resources can be found through the Substance Abuse and Mental Health Services Administration (www.samhsa.gov).

aMention of specific organizations does not constitute endorsement of these organizations by the authors

LGBTQIA+: lesbian, gay, bisexual, transgender, queer, intersex, and asexual; MSM: men who have sex with men; STI: sexual transmitted infection

Methamphetamine was the most commonly injected drug. Other drugs used for chemsex included ketamine, 3,4-methylenedioxymethamphetamine (MDMA, aka "ecstasy"), cocaine, amyl nitrite ("poppers"), and erectile dysfunction medications. ${ }^{1}$

It is important to remember that chemsex is a socially constructed concept and, as such, is subject to participant preferences and the popularity and availability of specific drugs. These features are likely to vary across geography, subcultures, and time. The above statistics ultimately represent a minority of MSM but highlight the importance of considering this phenomenon when caring for this population. ${ }^{1}$
When screening

for chemsex, approach patients in a curious, nonjudgmental, sex-positive manner 
To minimize the potential harms of chemsex, emphasize harm reduction rather than abstinence only

\section{What makes chemsex unique?}

Apps and access. Individuals who engage in chemsex report easy access to drugs via nightlife settings or through smartphone dating apps. Drugs are often shared during sexual encounters, which removes cost barriers for participants. ${ }^{1}$

Environment. Chemsex sometimes takes place in group settings at "sex-on-premises venues," including clubs, bathhouses, and saunas. The rise of smartphone apps and closure of these venues has shifted much of chemsex to private settings. ${ }^{1}$

Sexual behavior. Seventeen of the studies included in the Maxwell et $\mathrm{al}^{1}$ review showed an increased risk of condomless anal intercourse during chemsex. Several studies also reported increased rates of sex with multiple partners and new partners. ${ }^{1}$

\section{What are the potential risks?}

Physical health. High-risk sexual behaviors associated with chemsex increase the risk of sexually transmitted infections, including HIV and hepatitis C. ${ }^{1}$ Use of substances associated with chemsex can lead to overdose, cardiovascular events, and neurotoxicity. ${ }^{1,2}$

Mental health. In our clinical experience, the psychiatric implications of chemsex are numerous and exist on a spectrum from acute to chronic (Table 1, page 33).

\section{What can clinicians do?}

We encourage you to talk about chemsex with your patients. Table 2 (page 33) provides a "tip sheet" to help you start the conversation, address risks, and provide support. We hope you continue to learn from your patients and keep up-to-date on this evolving topic.

\footnotetext{
References

1. Maxwell S, Shahmanesh M, Gafos M. Chemsex behaviours among men who have sex with men: a systematic review of the literature. Int J Drug Policy. 2019;63:74-89.

2. Paulus MP, Stewart JL. Neurobiology, clinical presentation, and treatment of methamphetamine use disorder: a review. JAMA Psychiatry. 2020;77(9):959-966.
} 